\title{
DESEMPENHO REPRODUTIVO DE PORCAS SUBMETIDAS A INFUSÕES UTERINAS NO INÍCIO DO ESTRO'
}

\author{
FERNANDO PANDOLFO BORTOLOZZO ${ }^{2}$, IVO WENTZ ${ }^{3}$, GUILHERME BRANDT ${ }^{4}$ e ANTÔNIO LOURENÇO GUIDONI ${ }^{5}$
}

\begin{abstract}
RESUMO - O objetivo deste trabalho foi avaliar o desempenho reprodutivo de porcas multíparas submetidas à infusão uterina de diferentes soluções, realizada no início do estro. Um total de 1.019 fêmeas foram controladas no período de verão $(n=570)$ e inverno $(n=449)$. Os animais foram submetidos a cinco tratamentos, que consistiram em infusão de plasma seminal (PS), sêmen morto (SM), solução de 17ß-estradiol (SE), solução fisiológica (SS) e um grupo-controle (CO). As fêmeas receberam três inseminações: a primeira, 8-12 horas após a detecção do estro, e as demais, nos turnos subsequientes. Com relação à taxa de retorno ao estro e taxa de parto ajustada, não ocorreram diferenças entre os tratamentos $(\mathrm{p}>0,05)$. Na análise do modelo de regressão adotado para determinar o tamanho da leitegada, foi observada uma interação entre época do ano e tratamento $(\mathrm{p} \leq 0,01)$. No verão, as fêmeas que receberam infusão de PS apresentaram 0,89, 1,20, 1,34 e 2,31 leitões a mais, em relação aos tratamentos SE, SM, SS e CO ( $<<0,05)$, respectivamente. No inverno, o grupo submetido ao tratamento SS aumentou a produção em 1,25 e 0,91 leitões, respectivamente, em relação aos tratamentos SM e $\mathrm{CO}(\mathrm{p}<0,05)$. Este trabalho demonstrou que a infusão uterina de PS foi eficiente somente no verão, para aumentar o número de leitões nascidos. Entretanto, são necessários estudos complementares esclarecendo o efeito deste tratamento em épocas do ano distintas e em diferentes ordens de parto.
\end{abstract}

Termos para indexação: plasma seminal, sêmen morto, estradiol, taxa de parto, tamanho da leitegada.

\section{REPRODUCTIVE PERFORMANCE OF SOWS SUBMITTED TO UTERINE INFUSIONS IN THE BEGINNING OF OESTRUS}

\begin{abstract}
This study aimed to evaluate the reproductive performance of sows submitted to uterine infusion with different products at the beginning of oestrus. A total number of 1.019 females were controlled during the summer $(n=570)$ and winter $(n=449)$. Sows were submitted to five treatments, which consisted of infusions of seminal plasma (SP), dead semen (DS), 17 $\beta$-estradiol solution (ES), and physiological solution (PS) and a control group (CG). Sows received three inseminations, the first performed 8-12 hours after heat detection, and the other in the following morning and/or afternoon. In relation to return to oestrus rate and adjusted farrowing rate, there were no differences between treatments $(p>0.05)$. In the analysis of the regression model adopted to determine litter size, an interaction between season and treatment was observed $(\mathrm{p} \leq 0.01)$. In the summer, sows submitted to SP infusion had more $0.89,1.20,1.34$ and 2.31 piglets than those treated with ES, DS, PS and CG $(\mathrm{p}<0.05)$. In the winter, the group receiving PS increased litter size in 1.25 and 0.91 piglets compared to DS and CG $(\mathrm{p}<0.05)$. This study showed that uterine infusion of seminal plasma is efficient to increase the number of born piglets specially during the summer. However, complementary studies are needed to explain the effect of this treatment in different seasons and in different farrowing order.
\end{abstract}

Index terms: seminal plasma, dead semen, estradiol, farrowing rate, litter size.

${ }^{1}$ Aceito para publicação em 10 de maio de 1999.

${ }^{2}$ Méd. Vet., Dr., Prof. Adj., Faculdade de Veterinária, UFRGS, Av. Bento Gonçalves, 9090, CEP 90540-000 Porto Alegre, RS.E-mail: fpbortol@vortex.ufrgs.br

${ }^{3}$ Méd. Vet., Dr., Prof. Tit., Centro de Ciências Rurais, UFSM, Campus de Camobi, km 9, CEP 97105-900 Santa Maria, RS.

${ }^{4}$ Méd. Vet., M.Sc., Perdigão Agroindustrial S/A, Caixa Postal 20, CEP 89560-000 Videira, SC.

${ }^{5}$ Méd. Vet., Dr., Embrapa-Centro Nacional de Pesquisa de Suínos e Aves (CNPSA), Caixa Postal 21, CEP 89700-000 Concórdia, SC.

\section{INTRODUÇÃO}

Na espécie suína, a viabilidade dos gametas masculinos no trato genital feminino é limitada a um curto período de tempo. Portanto, o momento da inseminação, em relação à ovulação, é extremamente importante para a obtenção de altas taxas de fertilização (Waberski \& Weitze, 1996). O armazenamento dos espermatozóides na porção caudal do istmo por um período prolongado leva a perdas irreversíveis 
na capacidade de fertilização (Waberski, 1997). Essas perdas são mais pronunciadas nas chamadas espécies de ovulação espontânea, como a eqüina e a suína, cujo intervalo entre o início do estro e a ovulação pode se estender por vários dias (Waberski, 1997). Nesses casos é necessário realizar as inseminações repetidas vezes em um mesmo estro, para assegurar altos índices de fertilidade. Em virtude desse fato, na prática com suínos, o intervalo entre duas inseminações e entre a última inseminação e a ovulação não deve exceder 12 a 18 horas (Waberski \& Weitze, 1996).

Pitkjanen (1958) e Signoret et al. (1972) sugerem que a monta natural provoca uma redução do intervalo início do estro-ovulação. Acredita-se que esse fenômeno esteja relacionado a estímulos associados com a presença do macho (Kirsch et al., 1985), bem como a componentes específicos do sêmen do cachaço (Claus, 1989; Weitze et al., 1990a).

O plasma seminal desempenha um papel muito importante nos eventos fisiológicos necessários para se obter sucesso no desempenho reprodutivo (Claus, 1990; Weitze et al., 1990b). Quando infundido no útero no início do estro, leva a uma antecipação de 14,4 horas do momento da ovulação (Weitze et al., 1990a). Acredita-se que os estrógenos, presentes em altas concentrações no ejaculado (11,5 $\mu \mathrm{g} /$ ejaculado), promovem a liberação de prostaglandinas pelo endométrio, e estas, por sua vez, estimulam o transporte espermático passivo e induzem a ovulação (Claus et al., 1987, 1989; Claus, 1989). O plasma seminal também poderia influenciar o momento da ovulação antecipando o pico pré-ovulatório de LH (Claus, 1989). Recentemente, Waberski et al. (1995) mostraram a participação de uma fração peptídica de 1-10 kDa atuando de forma aditiva aos estrógenos seminais nesse processo.

O objetivo deste trabalho foi avaliar o desempenho reprodutivo de porcas submetidas a infusão uterina de plasma seminal, sêmen morto, solução de $17 \beta$-estradiol e solução fisiológica no início do estro da cobertura.

\section{MATERIAL E MÉTODOS}

O experimento foi realizado em um rebanho comercial de 3.400 matrizes, na Região Sul do Brasil (27 $15^{\prime}$ latitude
Sul e $52^{\circ} 1^{\prime}$ longitude Leste). Um total de 1.019 fêmeas híbridas Camborough $\mathrm{Ne}$ Camborough 15 foram controladas no período de verão $(n=570)$ e inverno $(n=449)$. $\mathrm{Na}$ formação dos grupos, as matrizes foram categorizadas, de acordo com a paridade $(\mathrm{P})$, em primíparas $(\mathrm{P}=1)$, fêmeas de segundo parto $(\mathrm{P}=2)$ e fêmeas com mais de dois partos $(\mathrm{P}>2)$, e de acordo com a linhagem. No desmame, as fêmeas foram classificadas de acordo com o escore corporal visual. O diagnóstico de estro foi realizado duas vezes ao dia ( 8 h30e 16h30), com o auxílio do macho, com o objetivo de determinar o início do período de apresentação do reflexo de tolerância à pressão lombar. Foram empregadas as matrizes que apresentaram um intervalo desmame-estro igual ou inferior a seis dias. No momento em que o estro foi detectado (hora zero) as fêmeas foram submetidas a infusão uterina de plasma seminal (PS), sêmen morto (SM), solução de 17ß-estradiol (SE), solução fisiológica (SS) ou mantidas no grupo-controle (CO).

As soluções de plasma seminal e de sêmem morto foram obtidas a partir de um pool de ejaculados mantidos a $-20^{\circ} \mathrm{C}$ até o processamento. Ao descongelar, a metade do pool foi utilizada para o tratamento SM, e a outra metade foi centrifugada a $1.500 \mathrm{~g}$ por 15 minutos, e o sobrenadante foi utilizado para o tratamento PS. A solução fisiológica foi preparada com $0,9 \%$ de cloreto de sódio. Essas soluções foram armazenadas em frascos de inseminação artificial em um volume de $100 \mathrm{~mL}$ e mantidas a $-20^{\circ} \mathrm{C}$. A solução de estradiol foi preparada a partir de uma solução-mãe com $10 \mathrm{mg}$ de $17 \beta$-estradiol ( $\beta$-estradiol 1,3,5[10]Estratriene-3,17 -diol - Sigma E-8875) dissolvidos em $100 \mathrm{~mL}$ de etanol e armazenada a $5^{\circ} \mathrm{C}$. Imediatamente antes da infusão pipetou-se $100 \mu \mathrm{L}$ desta solução em $100 \mathrm{~mL}$ de SS, portanto a SE continha $10 \mu \mathrm{g}$ de $17 \beta$-estradiol.

As fêmeas receberam três inseminações. A primeira foi realizada no turno seguinte (manhã ou tarde) ao qual havia sido detectado o início do estro, ou seja, pela manhã às $9 \mathrm{~h}$ ou à tarde às $17 \mathrm{~h}$. Nos dois turnos seguintes à primeira inseminação, as fêmeas receberam a segunda e a terceira dose, nos mesmos horários, de acordo com respectivos turnos. Cada dose inseminante empregada continha 4 bilhões de espermatozóides provenientes de um pool de três ejaculados. O diluente empregado foi o BTS (Pursel \& Johnson, 1975) sendo a dose armazenada a $15^{\circ} \mathrm{C}$ por, no máximo, 24 horas.

$\mathrm{O}$ experimento foi delineado em blocos inteiramente casualizados, em esquema fatorial $5 \times 2$, representados, respectivamente, pelos tratamentos e pela época do ano. A duração do estro e o tamanho da leitegada foram analisados pelo procedimento GLM do pacote estatístico SAS Institute (1985), e as médias foram comparadas pelo teste t. Para escolher as covariáveis, utilizou-se o procedimento 
STEPWISE. A única covariável estatisticamente significativa para a duração do estro foi o intervalo desmame-estro com $\mathrm{p}<0,01$. No entanto, para o tamanho da leitegada as covariáveis duração do estro $(\mathrm{p}=0,01)$, temperatura corporal da fêmea no dia da primeira cobertura $(\mathrm{p}=0,07)$, número médio de leitões nascidos nos partos anteriores $(\mathrm{p}<0,01)$, e escore corporal ao desmame $(\mathrm{p}=0,02)$ foram estatisticamente significativas.

A taxa de retorno ao estro foi calculada pela divisão do número de fêmeas que retornaram ao estro até 45 dias após a inseminação artificial pelo número de fêmeas submetidas a inseminação e multiplicado por 100. A taxa de parto foi calculada pela divisão do número de fêmeas paridas pelo número de fêmeas submetidas a inseminação e o resultado multiplicado por 100. A taxa de parto ajustada foi calculada a partir da taxa de parto, porém desconsiderando as fêmeas que apresentaram aborto e foram descartadas ou morreram (Dial et al., 1992).As variáveis taxa de retorno ao estro, taxa de parto e taxa de parto ajustada foram comparadas pelo Método de Comparação de Porcentagens Observadas, baseado na distribuição binomial (PimentelGomes, 1987). As médias das porcentagens observadas após submetidas à transformação arco seno $\sqrt{\% / 100}$ foram comparadas pelo teste $\mathrm{t}$, trabalhando-se com o número médio harmônico das repetições para cada fator ou sua combinação.

\section{RESULTADOS E DISCUSSÃO}

Um total de 1.019 matrizes inicialmente controladas no experimento foi utilizado somente para fins de análise da variável duração do estro. Quanto às demais análises, foram consideradas somente as matrizes que receberam três doses inseminantes. Com relação à taxa de retorno ao estro e à taxa de parto, utilizaram-se 892 fêmeas, ao passo que para a taxa de parto ajustada e tamanho das leitegadas utilizaramse, respectivamente, 855 e 778 matrizes. Durante a fase experimental o período médio de lactação foi $21,94 \pm 0,04$ dias, e o intervalo desmame-estro, de 4,58 $\pm 0,02$ dias.

Na Tabela 1 são apresentados os dados referentes à duração do estro, taxa de retorno ao estro, taxa de parto e taxa de parto ajustada. A duração do estro foi reduzida nas fêmeas submetidas à infusão uterina com plasma seminal. A diferença de 2,04 e 3,15 horas em relação às fêmeas tratadas com estradiol e as fêmeas do grupo-controle, respectivamente, foi estatisticamente significativa $(\mathrm{p}<0,05)$. As fêmeas tratadas com plasma seminal apresentaram uma taxa de retorno ao estro ligeiramente inferior às do grupocontrole, não sendo a diferença de $2,83 \%$, porém, estatisticamente significativa $(\mathrm{p}=0,19)$. As fêmeas tratadas com plasma seminal apresentaram uma taxa de parto 7,02\% superior $(\mathrm{p}=0,01)$ às do grupo-controle. Ao se analisar a taxa de parto ajustada, não foi observada diferença entre as fêmeas tratadas com plasma seminal e as do grupo-controle $(\mathrm{p}=0,13)$.

Foi observada uma redução na duração do estro das fêmeas submetidas à infusão de plasma seminal, em relação às tratadas com estradiol ou do grupocontrole. Sabe-se que existe uma correlação positiva entre a duração do estro e o intervalo início do estroovulação (Weitze et al., 1994; Soede et al., 1995; Heck et al., 1997; Niessen et al., 1997). Ou seja, as fêmeas que apresentam um estro longo apresentam um intervalo início do estro-ovulação maior que fêmeas com estro de curta duração. Dessa forma, pode-se especular que as fêmeas submetidas à infusão de plasma seminal, apresentando um estro em média de menor duração, também tiveram um menor intervalo entre o início do estro e a ovulação. A antecipação do momento da ovulação, descrita previamente por Weitze et al. (1990a) e Waberski (1997), não foi observada, no entanto, em experimentos mais recentes aplicando-se uma infusão uterina de plasma seminal em leitoas (Martini et al., 1997) ou aplicando PS em porcas tratadas com hCG (Soede et al., 1998). Ainda empregando o modelo Mariensee descrito por Waberski et al. (1995), Stahlberg et al. (1997) não observaram nenhuma variação significativa no momento da ovulação após a infusão uterina de PS no início do estro. A interação das secreções genitais do macho e do trato genital feminino, que poderiam levar à antecipação da ovulação que se segue à infusão uterina de plasma seminal no início do estro, tem o efeito mais pronunciado nas fêmeas que apresentam maior intervalo início do estro-ovulação e são submetidas à infusão mais próximas quanto possível do início do estro (Waberski, 1997). As variações de tais observações podem estar relacionadas ao PS, levando em conta a origem, técnica de armazenamento e preparo do mesmo. Da mesma forma, poderiam estar envolvidos aspectos relacionados ao genótipo das matrizes, à época do ano e também ao manejo reprodutivo do rebanho, levando em conta princi- 
palmente o momento no qual a infusão é realizada, à técnica de diagnóstico do estro e, consequientemente, ao intervalo início do estro-ovulação.

Os tratamentos não apresentaram efeito sobre a taxa de retorno ao estro. Em relação à taxa de parto, a superioridade aparente das fêmeas tratadas com PS em relação ao grupo CO foi nula, quando considerada a taxa de parto ajustada. Tal fato talvez possa ser explicado em virtude de as fêmeas terem recebido três doses inseminantes, sendo cada uma delas de 4 bilhões de espermatozóides. Niessen et al. (1997), realizando uma única inseminação com 2 bilhões de espermatozóides, obteve taxas de parto de $89 \%$ e $88 \%$, quando a inseminação foi realizada $28-25$ horas, 24 horas antes da ovulação, respectivamente. Da mesma forma, Waberski et al. (1996) acreditam que o plasma seminal não desempenhe papel importante no sucesso da fertilização em condições onde haja bom desempenho reprodutivo. Os autores alcançaram altas taxas de fertilização até mesmo após uma única inseminação com um baixo número de espermatozóides $\left(0,5 \times 10^{9}\right.$ espermatozóides).

Com relação ao tamanho da leitegada, foi demonstrado que, com uma infusão uterina de plasma seminal no início do estro, foi possível aumentar em até 1,37 leitões nascidos, em comparação com o grupocontrole. Flowers \& Esbenshade (1993), trabalhando com pré-infusões 20 minutos antes da cobertura, obtiveram taxas de parto significativamente melhores com o uso de plasma seminal. Porém, no seu trabalho, houve a associação das pré-infusões com o estímulo da monta. Os autores sugerem que os resultados obtidos após a realização de tratamentos pré-cobertura estarão mais evidentes quando se empregar a inseminação artificial isoladamente. Isto porque na monta natural, é possível que a fêmea receba os estímulos completos da cobertura propriamente dita. Deve-se considerar, contudo, que na inseminação artificial a concentração de estrógenos seminais e outros componentes do plasma seminal estão reduzidos e parcialmente substituídos pelos diluentes. Em trabalho semelhante ao de Flowers \& Esbenshade (1993), porém com leitoas e sem a associação da monta natural, Pozzobon et al. (1999) não encontraram resultados favoráveis sobre as taxas de retorno ao estro e taxas de parto com o uso de infusões com plasma seminal imediatamente antes da inseminação artificial. Outros trabalhos, entretanto, correlacionam a utilização de pré-infusões com melhoras na taxa de fertilização e no transporte espermático, o que pode ser refletido no desempenho reprodutivo (Peña-Alfaro, 1988; Willmen, 1989; Rabeler, 1990).

Segundo Claus (1990), juntamente com os espermatozóides, outros componentes do processo da cobertura são requeridos para a otimização das taxas de parto e tamanho da leitegada. Muitos dos estímulos associados com a cobertura propriamente dita influenciam importantes eventos fisiológicos necessários para o sucesso da reprodução.

A redução do período do estro e eventual antecipação da ovulação ocorrida após a infusão uterina com plasma seminal podem ter sido o fator diferenciador dos resultados relacionados ao tamanho da leitegada. Entretanto, no presente trabalho, por não ter sido determinado o momento da ovulação, não foi possível confirmar tais especulações.

TABELA 1. Efeito dos tratamentos sobre a duração do estro, taxa de retorno ao estro, taxa de parto e taxa de parto ajustada ${ }^{1}$.

\begin{tabular}{lccccc}
\hline Tratamento $^{2}$ & $\begin{array}{c}\text { Matrizes } \\
(\mathrm{n})\end{array}$ & $\begin{array}{c}\text { Duração do estro } \\
\text { (horas) }\end{array}$ & $\begin{array}{c}\text { Taxa de retorno } \\
\text { ao estro }(\%)\end{array}$ & $\begin{array}{c}\text { Taxa de parto } \\
(\%)\end{array}$ & $\begin{array}{c}\text { Taxa de parto } \\
\text { ajustada }(\%)\end{array}$ \\
\hline PS & 223 & $48,93 \pm 0,73 \mathrm{a}$ & $7,54 \pm 0,01 \mathrm{a}$ & $89,95 \pm 0,01 \mathrm{a}$ & $92,27 \pm 0,01 \mathrm{a}$ \\
SE & 202 & $50,97 \pm 0,73 \mathrm{bc}$ & $7,73 \pm 0,01 \mathrm{a}$ & $87,85 \pm 0,01 \mathrm{ab}$ & $91,91 \pm 0,01 \mathrm{a}$ \\
SM & 203 & $49,82 \pm 0,73 \mathrm{ab}$ & $8,43 \pm 0,01 \mathrm{a}$ & $87,64 \pm 0,01 \mathrm{ab}$ & $91,23 \pm 0,01 \mathrm{a}$ \\
SS & 196 & $49,63 \pm 0,73 \mathrm{ab}$ & $9,41 \pm 0,01 \mathrm{a}$ & $87,06 \pm 0,01 \mathrm{ab}$ & $90,24 \pm 0,01 \mathrm{a}$ \\
CO & 195 & $52,08 \pm 0,73 \mathrm{c}$ & $10,37 \pm 0,01 \mathrm{a}$ & $82,93 \pm 0,01 \mathrm{~b}$ & $88,89 \pm 0,01 \mathrm{a}$ \\
\hline
\end{tabular}

${ }^{1}$ Médias seguidas por letras iguais na coluna não diferem $(\mathrm{p}>0,05)$ pelo teste $\mathrm{t}$.

2 PS: plasma seminal; SE: solução de 17ß-estradiol; SM: sêmen morto; SS: solução fisiológica; CO: controle. 
Apesar de apresentar vantagens práticas para sua preparação, a utilização de sêmen morto não apresentou resultados favoráveis à eficiência reprodutiva. Segundo Hunter (1988), a presença de anticorpos contra espermatozóides ou mesmo proteínas do plasma seminal, no lúmen do trato genital feminino, podem produzir aglutinações de um grande número de espermatozóides capazes de provocar sua aderência ao endométrio (Baker \& Degen, 1972), o que, posteriormente, poderia comprometer a viabilidade dos espermatozóides na inseminação artificial.

$\mathrm{Na}$ análise do modelo de regressão adotado para determinação do tamanho da leitegada, foi possível observar uma interação significativa de tratamento com época do ano $(\mathrm{p}=0,01)$. No período de verão, as fêmeas tratadas com plasma seminal apresentaram um acréscimo no número de leitões nascidos, em relação às demais (Tabela 2). No inverno, a superioridade foi do grupo tratado com soro fisiológico em relação ao grupo tratado com sêmen morto ou ao grupo-controle, porém não foram estatisticamente diferentes das fêmeas que receberam os tratamentos com plasma seminal e estradiol. Durante o verão, o tratamento com plasma seminal proporcionou um aumento de 0,89, 1,2, 1,34 e 2,31 leitões em relação aos tratamentos SE, SM, SS e CO, respectivamente; essa diferença foi significativa estatisticamente $(\mathrm{p}<0,05)$. No inverno, o grupo tratado com soro fisiológico

TABELA 2. Efeito dos tratamentos nos períodos de verão e inverno sobre o tamanho da leitegada (número de leitões \pm desviopadrão) ${ }^{1}$.

\begin{tabular}{cccccc}
\hline Tratamento $^{2}$ & \multicolumn{2}{c}{ Verão } & & \multicolumn{2}{c}{ Inverno } \\
\cline { 2 - 3 } \cline { 5 - 6 } & $\begin{array}{c}\text { Matrizes } \\
(\mathrm{n})\end{array}$ & $\begin{array}{c}\text { Leitões } \\
(\mathrm{n} \pm \mathrm{DP})\end{array}$ & & $\begin{array}{c}\text { Matrizes } \\
(\mathrm{n})\end{array}$ & $\begin{array}{c}\text { Leitões } \\
(\mathrm{n} \pm \mathrm{DP})\end{array}$ \\
\hline $\mathrm{PS}$ & 98 & $12,72 \pm 0,32 \mathrm{a}$ & 81 & $11,86 \pm 0,31 \mathrm{ab}$ \\
$\mathrm{SE}$ & 93 & $11,83 \pm 0,32 \mathrm{~b}$ & 66 & $11,85 \pm 0,32 \mathrm{ab}$ \\
$\mathrm{SM}$ & 84 & $11,52 \pm 0,32 \mathrm{~b}$ & 70 & $11,09 \pm 0,31 \mathrm{~b}$ \\
$\mathrm{SS}$ & 78 & $11,38 \pm 0,32 \mathrm{~b}$ & 72 & $12,34 \pm 0,32 \mathrm{a}$ \\
$\mathrm{CO}$ & 68 & $10,41 \pm 0,32 \mathrm{c}$ & 68 & $11,43 \pm 0,31 \mathrm{~b}$ \\
\hline
\end{tabular}

1 Médias seguidas por letras iguais na coluna não diferem $(p>0,05)$ pelo teste $\mathrm{t}$.

2 PS: plasma seminal; SE: solução de 17ß-estradiol; SM: sêmen morto; SS: solução fisiológica; $\mathrm{CO}$ : controle. aumentou a produção em 1,25 e 0,91 leitões, em relação aos tratamentos sêmen morto e controle, respectivamente, com uma diferença estatisticamente significativa $(\mathrm{p}<0,05)$.

O presente trabalho demonstrou que a infusão uterina com plasma seminal pré-cobertura, aumentou o número de leitões nascidos no verão, período considerado crítico à eficiência reprodutiva do rebanho; no inverno, igualou-se às demais. Entretanto, para recomendações estratégicas no uso dessa tecnologia, são necessários estudos complementares esclarecendo o efeito desse tratamento em épocas do ano distintas e em diferentes ordens de parto.

\section{CONCLUSÃO}

A infusão uterina de plasma seminal pré-cobertura, no período de verão, é eficiente para aumentar o tamanho da leitegada.

\section{REFERÊNCIAS}

BAKER, R.D.; DEGEN, A.A. Transport of live and dead boar spermatozoa within the reproductive tract of gilts. Journal of Reproduction and Fertility, Delhi, v.28, p.369-377, 1972.

CLAUS, R. Oestrogens of the boar: effects on male and female reproductive functions. In: HOLSTEIN, A.F.; VOIGT, K.D.; GRÄSSLIN, D. (Eds.). Reproductive biology and medicine. Diesbach : Diesbach Verlag, 1989. p.136-147.

CLAUS, R. Physiological role of seminal components in the reproductive tract of the female pig. Journal of Reproduction and Fertility, Delhi, v.40, p.117-131, 1990. Supplement.

CLAUS, R.; ELLENDORFF, F.; HOANG-VU, C. Spontaneous electromyographic activity throughout the cycle in the sow and its change by intrauterine oestrogen infusion during oestrus. Journal of Reproduction and Fertility, Delhi, v.87, p.543-551, 1989.

CLAUS, R.; HOANG-VU, C.; ELLENDORFF, F.; MEYER, H.D.; SCHOPPER, D.; WEILLER, U. Seminal oestrogens of the boar: origin and functions in the sow. Journal of Steroid Biochemistry, Elmsford, v.27, p.331-335, 1987.

Pesq. agropec. bras., Brasília, v.35, n.3, p.623-629, mar. 2000 
DIAL, G.D.; MARSH, W.E.; POLSON, D.D.; VAILLANCOURT, J.-P. Reproduction failure: differential diagnosis. In: LEMAN, A.D.; STRAW, B.E.; MENGELING, W.L.; ALLAIRE, S.D.; TAYLOR, D.J. (Eds.). Diseases of swine. Ames : Iowa State University Press, 1992. p.88-137.

FLOWERS, W.L.; ESBENSHADE, K.L. Optimizing management of natural and artificial matings in swine. Journal of Reproduction and Fertility, Delhi, v.48, p.217-228, 1993. Supplement.

HECK, A.; BORTOLOZZO, F.P.; WENTZ, I.; MARTINI, R.L.; STAHLBERG, R.; GUIDONI, A.L.; NAGAE, R. Determinação do momento da ovulação em porcas de granjas comerciais via diagnóstico ultra-sonográfico transcutâneo. In: CONGRESSO BRASILEIRO DE VETERINÁRIOS ESPECIALISTAS EM SUÍNOS, 8., 1997, Foz do Iguaçu. Anais. Foz do Iguaçu : ABRAVES, 1997. p.333-335.

HUNTER, R.H.F. The fallopian tubes. Berlin : Springer, 1988.191p.

KIRSCH, J.D.; TILTON, J.E.; ZIECIK, A.; WEIGL, R.; SCHAFFER, T.; WILLIAMS, G.L. Effect of various mating stimuli on pituitary release of luteinizing hormone in the gilt. Domestic Animal Endocrinology, Auburn, v.2, p.99-105, 1985.

MARTINI, R.; WENTZ, I.; BORTOLOZZO, F.P.; HECK, A.; STAHLBERG, R.; UEMOTO, D.A.; NAGAE, R.; GUIDONI, A.L. Infusões uterinas de plasma seminal no início do estro e sua influência na eficiência reprodutiva de leitoas. In: CONGRESSO BRASILEIRO DE VETERINÁRIOS ESPECIALISTAS EM SUÍNOS, 8., 1997, Foz do Iguaçu. Anais. Foz do Iguaçu : ABRAVES, 1997. p.317-319.

NIESSEN, A.K.; SOEDE, N.M.; HYTTEL, P.; SCHMIDT, M.; D'HOORE, L. The influence of time of insemination relative to time of ovulation on farrowing frequency and litter size in sows, as investigated by ultrasonography. Theriogenology, Gainesville, v.41, p.1571-1582, 1997.

PEÑA-ALFARO, C.E. Einfluss des Seminalplasmas im Inseminant auf Spermentransport und Befruchtungserfolg bei Jungsauen unter besonderer Berücksichtigung unterschiedlicher Spermendosierungen in der flüssigkonservierten Samenportion. Hannover : Tierärztliche Hochschule Hannover, 1988. 113p. Doktorarbeit These.
PIMENTEL-GOMES, F. Curso de Estatística Experimental. 12.ed. Piracicaba : USP-ESALQ, 1987. p.383-401.

PITKJANEN, I.G. The time of ovulation in sows. Svinovodstvo, Moscow, v.12, p.38-40, 1958.

POZZOBON, M.C.; BORTOLOZZO, F.P.; WENTZ, I.; BOCHARDT NETO, G.; FLORES, L.A.S.; KOPPERCHIMIDT, P.; WABERSKI, D.; WEITZE, K.F.; PASQUAL, C. Parâmetros reprodutivos de fêmeas suínas submetidas à infusão trancervical de plasma seminal no início do estro. In: CONGRESSO BRASILEIRO DE VETERINÁRIOS ESPECIALISTAS EM SUÍNOS, 9., Belo Horizonte. Anais. Belo Horizonte : ABRAVES, 1999. p.373-374.

PURSEL, V.G.; JOHNSON, L.A. Freezing of boar spermatozoa, fertilizing capacity with concentrate semen and a new thawing procedure. Journal of Animal Science, Champaign, v.40, p.99-102, 1975.

RABELER, H.J. Einfluß von Seminalplasmainhaltsstoffen im Inseminat auf Befruchtungsraten, Spermentransport und Ovulation bei der Sau. Hannover : Tierärztliche Hochschule Hannover, 1990. 85p. Doktorarbeit These.

SAS INSTITUTE. SAS users guide: Statistics. 5.ed. Cary, NC, 1985. 956p.

SIGNORET, J.P.; DU BUISSON, F.M.; MAULÉON, P. Effect of mating on the onset and duration of ovulation in the sow. Journal of Reproduction and Fertility, Delhi, v.31, p.327-330, 1972.

SOEDE, N.M.; BOUWMAN, E.G.; KEMP, B. Seminal plasma does not advance ovulation in hCG-treated sows. Animal Reproduction Science, v.54, p.2329, 1998.

SOEDE, N.M.; WETZELS, C.C.H.; ZONDAG, W.; KONING, M.A.I.; KEMP, B. Effects of time of insemination relative to ovulation, as determined by ultrasonography, on fertilization rate and accessory sperm count in sows. Journal of Reproduction Fertility, Delhi, v.104, p.99-106, 1995.

STAHLBERG, R.; WENTZ, I.; BORTOLOZZO, F.P.; MARTINI, R.L.; HECK, A.; NAGAE, R.; MACHADO, I.P. Uso de modelo cirúrgico para o controle do momento da ovulação. In: CONGRESSO BRASILEIRO DE VETERINÁRIOS ESPECIALISTAS EM SUÍNOS, 8., 1997, Foz do Iguaçu. Anais. Foz do Iguaçu : ABRAVES, 1997. p.335-337. 
WABERSKI, D. Effects of semen components on ovulation and fertilization, Journal of Reproduction Fertility, Delhi, v.52, p.105-109, 1997. Supplement.

WABERSKI, D.; SOARES, J.A.G.; ARRUDA, E.B de; WEITZE, K.F. Effect of a transcervical infusion of seminal plasma prior to insemination on the fertilising competence of low numbers of boar spermatozoa at controlled AI-ovulation intervals. Animal Reproduction Science, Amsterdam v.44, p.165-173, 1996.

WABERSKI，D.; SUEDHOFF，H.; HAHN，T.; JUNGBLUT, P.W.; KALLWEIT, E.; CALVETE, J.J.; ENSSLIN, M.; HOPPEN, H.O.; WINTERGALEN, N.; WEITZE, K.F.; TOEPFERPETERSEN, E. Advanced ovulation in gilts by the intrauterine application of a low molecular mass pronase-sensitive fraction of boar seminal plasma. Journal of Reproduction Fertility, Delhi, v.105, p.247-252, 1995.

WABERSKI, D.; WEITZE, K.F. Correct timing of artificial insemination in pigs. Reproduction in Domestic Animals, v.31, p.527-530, 1996.
WEITZE, K.F.; LOTZ, J.; EVERWAND, A.; WILLMEN, T.; WABERSKI, D. Interaction between inseminate, uterine and ovarian function in the sow. II. Investigations into the influencing of ovulation by the use of sperm free media. Reproduction in Domestic Animals, Berlin, v.25, p.197-204, 1990a.

WEITZE, K.F.; RATH, D.; WILLMEN, T.; WABERSKI, D.; LOTZ, J. Advancement of ovulation in the sow related to seminal plasma application before insemination. Reproduction in Domestic Animals, Berlin, v.25, p.61-67, 1990b.

WEITZE, K.F.; WAGNER-RITSCHEL, H.; WABERSKI, D.; RICHTER, L.; KRIETER, J. The onset of heat after weaning, heat duration, and ovulation as major factors in AI timing in sows. Reproduction in Domestic Animals, Berlin, v.29, p.433-443, 1994

WILLMEN, T. Einfluß von Spermendosierung und Seminalplasma im Inseminat auf Befruchtungsrate, Spermentransport und Ovulation beim Schwein. Hannover : Tierärztliche Hochschule Hannover, 1989. 95p. Doktorarbeit These. 